Supporting Information for

\title{
Comprehensive Characterization of Biotherapeutics by Selective Capturing of Highly Acidic Glycans using Stepwise PGC-SPE and LC/MS/MS
}

\author{
Youngsuk Seo ${ }^{1,2}$, Myung Jin $\mathrm{Oh}^{1,2}$, Jin Young Park ${ }^{1,2}$, \\ Jae Kyoung $\mathrm{Ko}^{1,2}$, Jin Young $\mathrm{Kim}^{3}$, and Hyun Joo $\mathrm{An}^{1,2, *}$
}

1. Asia Glycomics Reference Site, Daejeon 34134, Korea

2. Graduate School of Analytical Science and Technology, Chungnam National University, Daejeon 34134, Korea

3. Department of Mass Spectrometry, Korea Basic Science Institute, Ochang 28119, Korea

${ }^{*}$ To whom correspondence should be addressed:

Hyun Joo An

99 Daehak-ro, Yuseong-gu, Daejeon 34134, South Korea

Email: hjan@cnu.ac.kr 


\section{TABLE OF CONTENTS:}

Figure S-1. Representative extracted compound chromatogram of N-glycans released from a therapeutic lysosomal enzyme

Figure S-2. Extracted compound chromatograms of N-glycans analyzed by different LC/MS conditions

Figure S-3. The quantitative distribution of glycan groups of a therapeutic lysosomal enzyme Figure S-4. The charge states of phosphorylated glycans in SPE solutions at different $\mathrm{pH}$ values

Figure S-5. Experimental and technical reproducibility of the combination of PGC-SPE and LC-MS

Figure S-6. Pairwise comparisons of glycosylation of different batch enzymes

Table S-1. Total glycans of therapeutic lysosomal enzymes found in three SPE fractions 


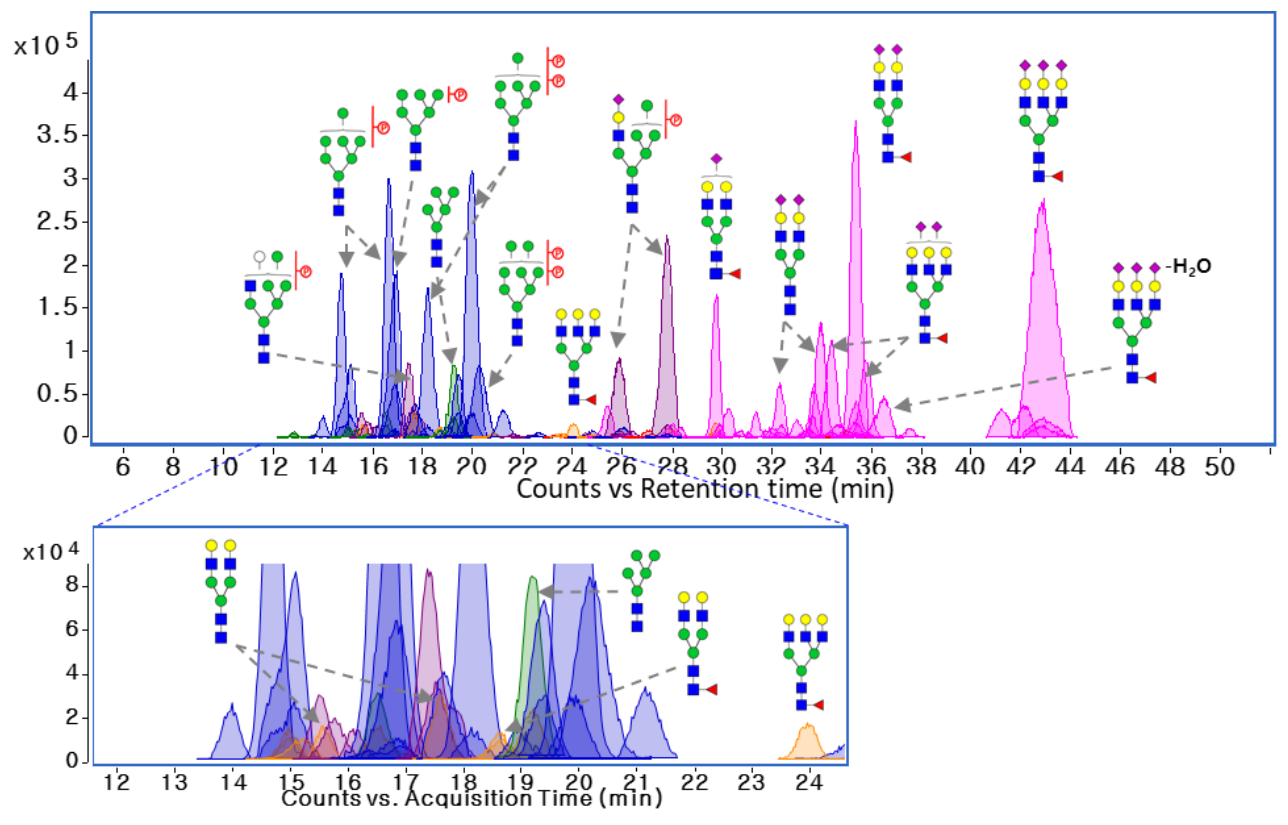

Figure S-1. Representative extracted compound chromatogram of N-glycans released from a therapeutic lysosomal enzyme. All glycans released from a therapeutic enzyme were separated by PGC chromatography and detected using high resolution Q-TOF MS. The chromatogram is differently colored according to biosynthetic class and chemical properties of glycans; green for neutral high-mannose, yellow for neutral complex, blue for phosphorylated high-mannose, purple for phosphorylated hybrid, and pink for sialylated complex/hybrid. A magnified view of the ECC (retention time 12-24 min) showed lowly abundant neutral glycans, which were co-eluted with phosphorylated glycans. 
(a)

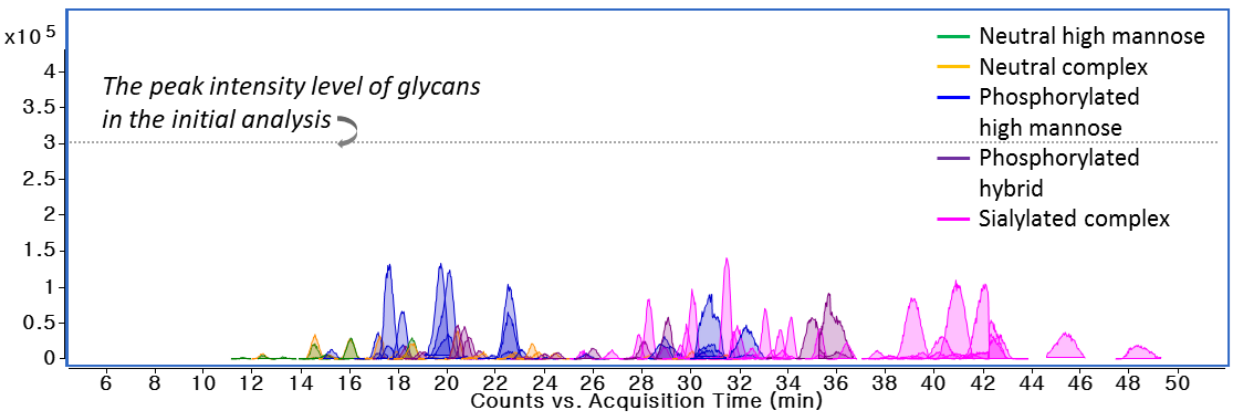

(b)

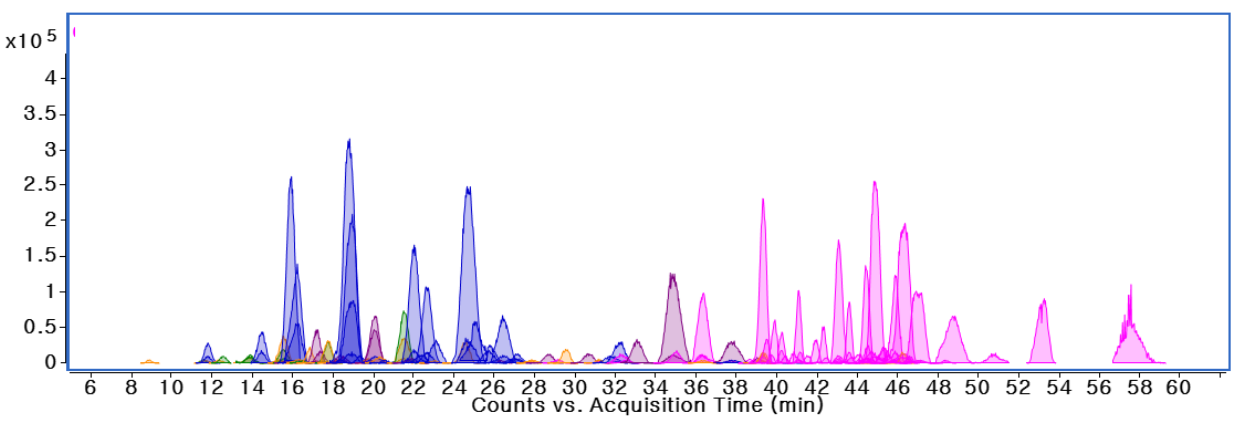

Figure S-2. Extracted compound chromatograms of N-glycans analyzed by different LC/MS conditions. Initially, N-glycans of a therapeutic lysosomal enzyme were separated via PGC column under the following LC conditions; (1) A solvent, 3\% ACN in $\mathrm{H}_{2} \mathrm{O}$ with $0.5 \%$ formic acid (FA) and B solvent, $90 \% \mathrm{ACN}$ in $\mathrm{H}_{2} \mathrm{O}$ with $0.5 \% \mathrm{FA}$; (2) in the gradient of 5-16\% B solvent in $6-25 \mathrm{~min}, 16-40 \% \mathrm{~B}$ in $25-45 \mathrm{~min}$, and $40-100 \% \mathrm{~B}$ in $45-50 \mathrm{~min}$. We altered LC/MS conditions to enhance peak resolutions of glycans (a) Different LC solutions. A solvent: 3\% $\mathrm{ACN}$ in $\mathrm{H}_{2} \mathrm{O}$, B solvent : $90 \% \mathrm{ACN}$ in $\mathrm{H}_{2} \mathrm{O}$ with $0.5 \%$ FA. (b) Different $\mathrm{LC}$ gradient of 5$16 \%$ B solvent in 6-35 min, $16-40 \% \mathrm{~B}$ in $35-55 \mathrm{~min}$, and $40-100 \% \mathrm{~B}$ in $55-60 \mathrm{~min}$. Both conditions cannot solve the separation problem of co-eluted glycans with different chemical properties. In addition, the reduction of acid in LC solvents resulted in insufficient ionization of glycans. 


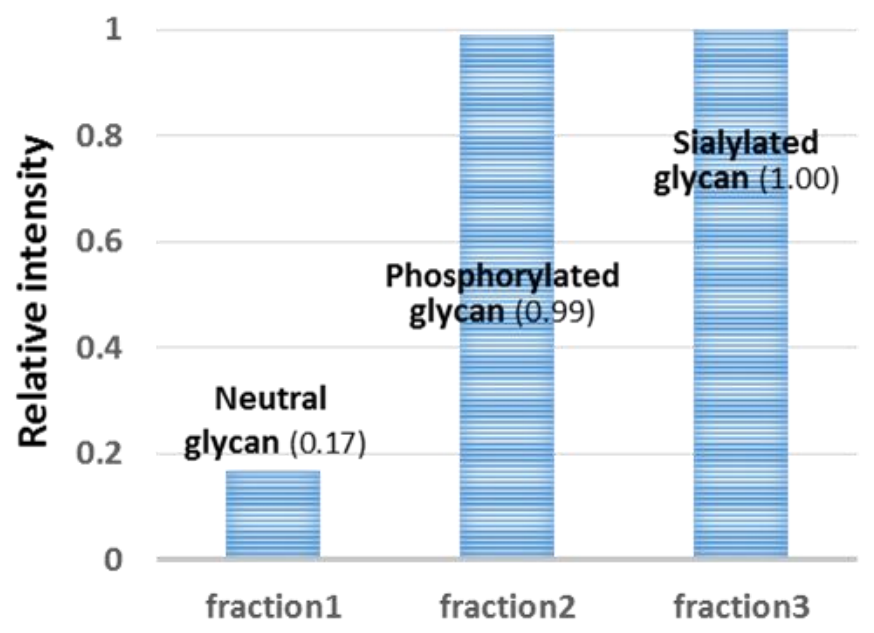

Figure S-3. The quantitative distribution of glycan groups of a therapeutic lysosomal enzyme. Neutral, phosphorylated, and sialylated glycan group were found in three SPE fractions, respectively. Peak intensities in ECCs of three glycan groups were independently summed. They were divided into the summed value of peak intensities of sialylated glycans. 


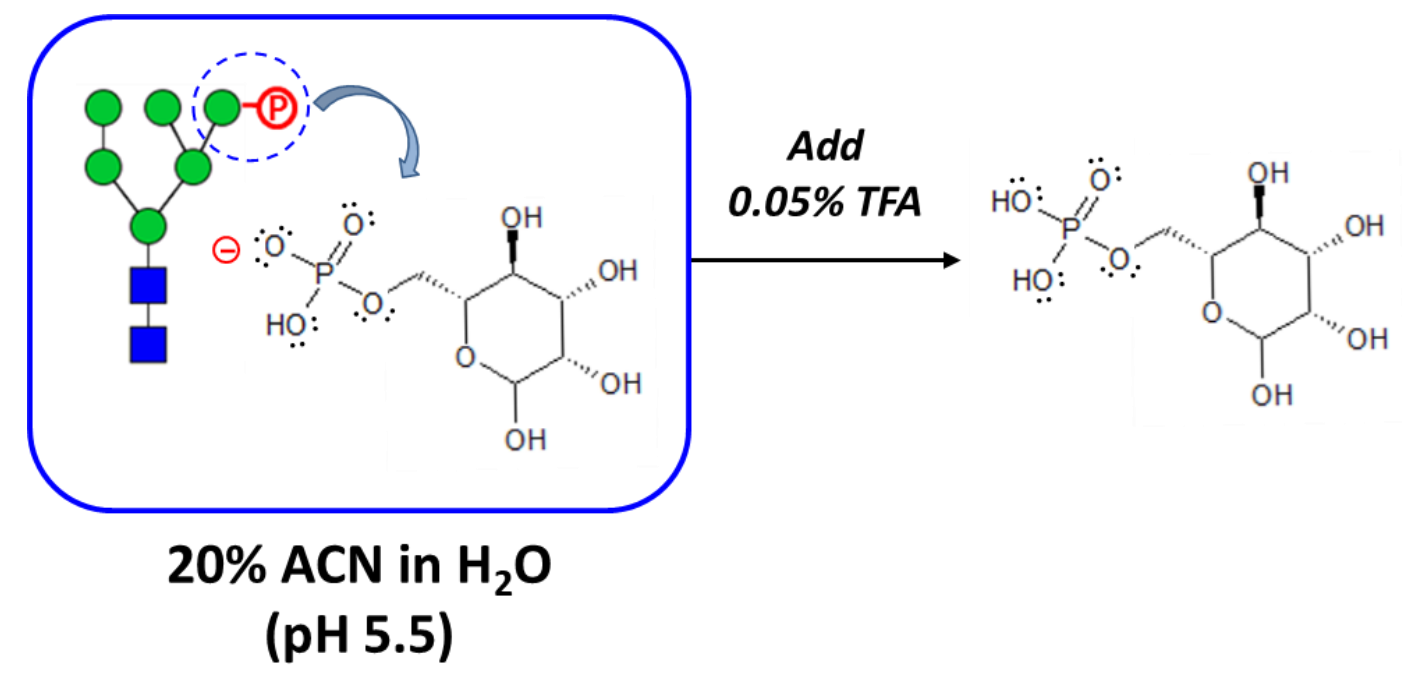

Figure S-4. The charge states of phosphorylated glycans in SPE solutions at different pH values. The phosphate group of a glycan was deprotonated in the SPE solution condition, 20\% ACN in $\mathrm{H}_{2} \mathrm{O}$ (pH 5.5), while it was protonated again by $0.05 \%$ TFA-containing solution ( $\mathrm{pH}$ 2.0) 
(a)

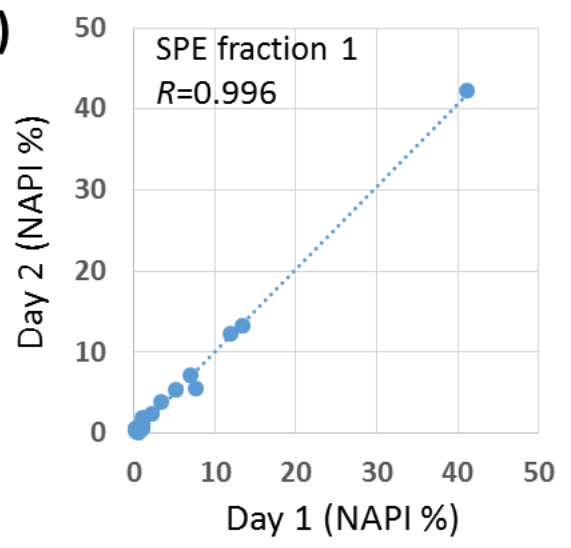

(c)

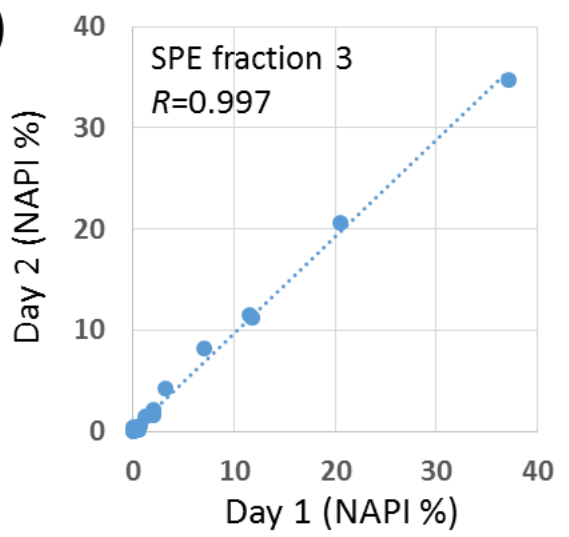

(b)

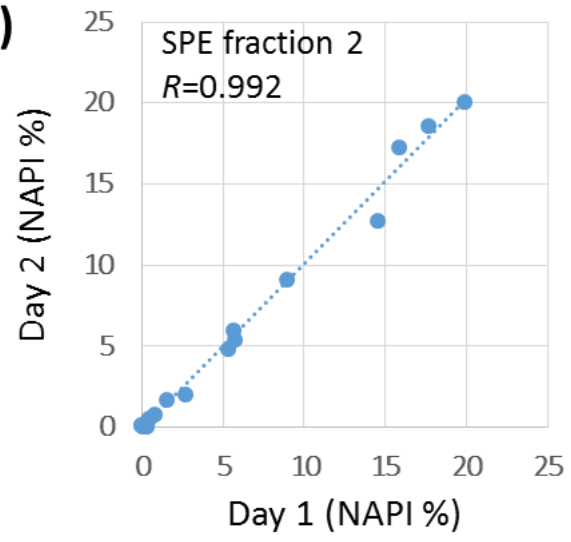

Figure S-5. Experimental and technical reproducibility of the combination of PGC-SPE and LC-MS. Using our analytical method, $\mathrm{N}$-glycans of a therapeutic lysosomal enzyme were obtained repeatedly on different two days and analyzed in triplicate. (a) Neutral glycan fractions by $20 \% \mathrm{ACN}$ in $\mathrm{H}_{2} \mathrm{O}$, (b) phosphorylated glycan fractions by $10 \% \mathrm{ACN}$ in $\mathrm{H}_{2} \mathrm{O}$ with acid, and (c) sialylated glycan fractions by $40 \% \mathrm{ACN}$ in $\mathrm{H}_{2} \mathrm{O}$ were pairwise compared using the NAPIs of single glycan species. R: Pearson correlation coefficient. 
(a)

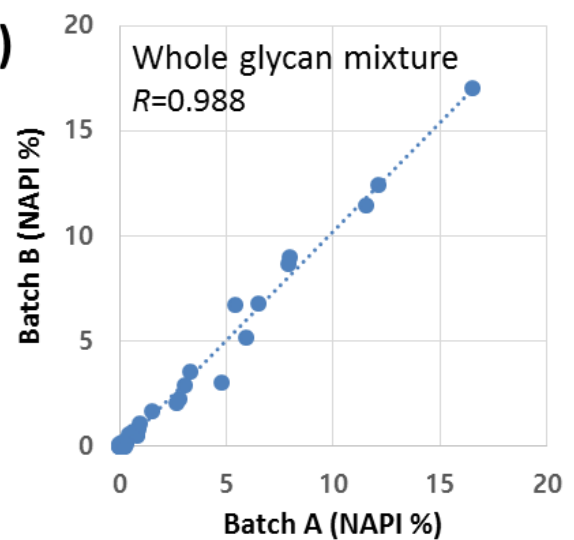

(c)

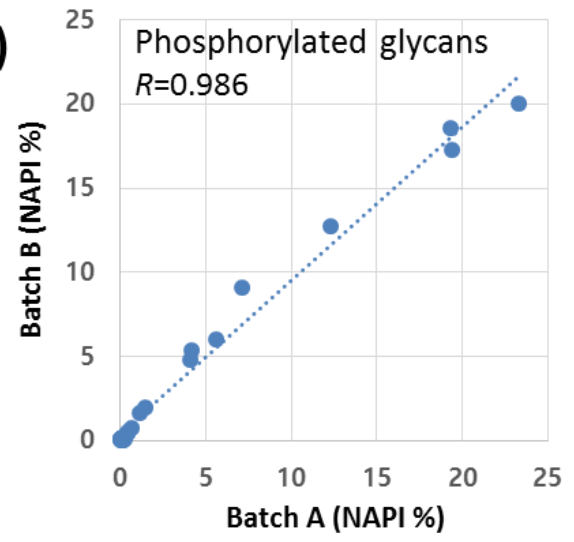

(b)

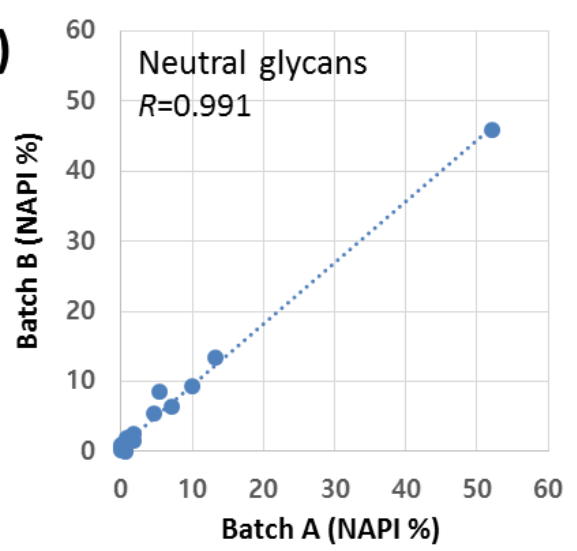

(d)

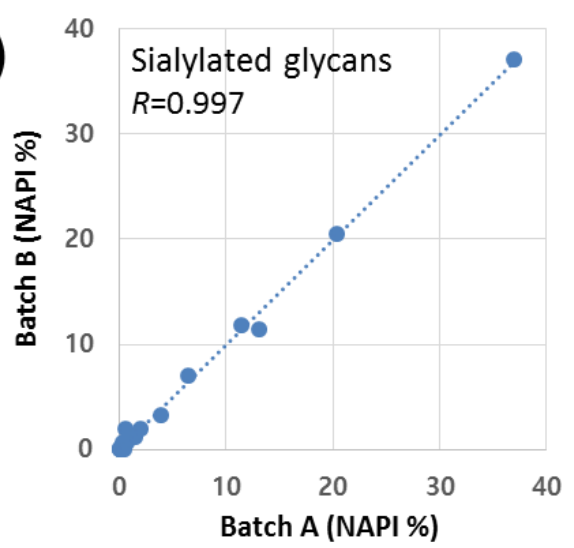

Figure S-6. Pairwise comparisons of glycosylation of different batch enzymes. (a) Whole glycan mixtures were obtained without selective fractionation from two batch products, respectively, and analyzed by PGC-LC-MS analysis. Three glycan groups, namely (b) neutral, (c) phosphorylated, and (d) sialylated glycan group, were obtained via the stepwise PGC-SPE. Two batches are compared based on fractionated glycan groups. Each point in the graphs represents single glycan species, with $\mathrm{x}$-and y-coordinates taken from NAPIs in different two batches. R: Pearson correlation coefficient. NAPI: Normalized absolute peak intensity. 
Table S-1. Total glycans of therapeutic lysosomal enzymes found in three SPE fractions

\begin{tabular}{|c|c|c|c|c|c|c|c|c|}
\hline \multicolumn{9}{|c|}{ The first eluent by $20 \% \mathrm{ACN}$ in $\mathrm{H}_{2} \mathrm{O}^{a}$} \\
\hline \multirow[b]{2}{*}{ Mass } & \multirow[b]{2}{*}{$\mathrm{Cpd}^{\mathrm{b}}$} & \multirow[b]{2}{*}{ Glycan type $^{c}$} & \multicolumn{3}{|c|}{$\begin{array}{c}\text { Batch A } \\
\text { Relative intensity }\end{array}$} & \multicolumn{3}{|c|}{$\begin{array}{c}\text { Batch B } \\
\text { Relative intensity }\end{array}$} \\
\hline & & & $\operatorname{Avg}^{d}$ & $\mathrm{SE}^{\mathrm{e}}$ & $\begin{array}{c}\text { Newly } \\
\text { ID }^{f}\end{array}$ & $\operatorname{Avg}^{d}$ & $\mathrm{SE}^{\mathrm{e}}$ & $\begin{array}{l}\text { Newly } \\
\text { ID }^{f}\end{array}$ \\
\hline 910.3278 & 3_2_0_0_0_0 & $\mathrm{HM}$ & 7.13 & 0.44 & & 6.48 & 0.19 & \\
\hline 1072.3806 & 4_2_0_0_0_0 & $\mathrm{HM}$ & 13.24 & 0.42 & & 13.47 & 1.34 & \\
\hline 1234.4334 & 5_2_0_0_0_0 & $\mathrm{HM}$ & 52.17 & 0.43 & & 45.77 & 1.74 & \\
\hline 1396.4863 & 6_2 $20 \_0 \_0 \_0$ & $\mathrm{HM}$ & 9.97 & 0.33 & & 9.25 & 0.36 & \\
\hline 1558.5391 & 7_2_0_0_0_0 & $\mathrm{HM}$ & 1.68 & 0.34 & & 2.51 & 0.17 & \\
\hline 1720.5919 & 8_2 $20-0 \_0 \_0$ & $\mathrm{HM}$ & 0.53 & 0.07 & 0 & 0.55 & 0.14 & 0 \\
\hline 1882.6447 & 9_2 $20 \_0 \_0 \_0$ & $\mathrm{HM}$ & - & - & & 0.23 & 0.03 & 0 \\
\hline 1437.5128 & 5_3_0_0_0_0 & $\mathrm{H}$ & 0.68 & 0.09 & 0 & 0.53 & 0.04 & 0 \\
\hline 1599.5656 & 6_3_0_0_0_0 & $\mathrm{H}$ & 1.69 & 0.18 & & 1.48 & 0.04 & \\
\hline 1056.3857 & 3_2_1_0_0_0 & C & 0.62 & 0.13 & & 1.13 & 0.08 & 0 \\
\hline 1113.4072 & 3_3 $30 \_0 \_0 \_0$ & C & 0.39 & 0.13 & 0 & 0.40 & 0.10 & 0 \\
\hline 1259.4651 & 3_3_1_0_0_0 & C & - & - & & 0.68 & 0.13 & 0 \\
\hline 1316.4865 & 3_4_0_0_0_0 & C & 0.64 & 0.15 & 0 & - & - & \\
\hline 1421.5179 & 4_3_1_0_0_0 & C & - & - & & 0.36 & 0.12 & \\
\hline 1478.5393 & 4_4_0_0_0_0 & C & - & - & & 0.94 & 0.14 & 0 \\
\hline 1624.5973 & 4_4_1_0_0_0 & C & 0.53 & 0.03 & 0 & 0.29 & 0.06 & 0 \\
\hline 1640.5922 & 5_4_O_O_O_O & C & 4.60 & 0.38 & & 5.51 & 0.74 & \\
\hline 1786.6501 & 5_4_1_0_0_0 & C & 5.32 & 0.35 & & 8.49 & 0.37 & \\
\hline 2151.7823 & 6_5_1_0_0_0 & $C$ & 0.82 & 0.18 & & 1.93 & 0.01 & 0 \\
\hline
\end{tabular}

a All glycans found in each eluent were listed

bGlycan composition, Hex_HexNAc_Fuc_NeuAc_NeuGc_Phosphate group Hex=mannose, galactose; $\mathrm{HexNAc}=\mathrm{N}$-acetylglucosamine; Fuc = fucse; NeuAc $=N$-acetyl neuraminic acid $;$ NeuGc $=N$-glycolyl neuraminic acid. ${ }^{\mathrm{C}} \mathrm{Glycan}$ types, $\mathrm{HM}=$ high mannose type; $\mathrm{H}=$ hybrid type; $\mathrm{C}=$ complex type

${ }^{\mathrm{d}}$ Avg $=$ Average

e $\mathrm{SE}=$ Standard error

${ }^{\mathrm{f}}$ Newly identified glycans by the combination of PGC-SPE and LC-MS analysis 


\begin{tabular}{|c|c|c|c|c|c|c|c|c|}
\hline \multicolumn{9}{|c|}{ The second eluent by $10 \% \mathrm{ACN}$ in $\mathrm{H}_{2} \mathrm{O}$ with $0.05 \% \mathrm{TFA}^{\mathrm{a}}$} \\
\hline \multirow[b]{2}{*}{ Mass } & \multirow[b]{2}{*}{$\mathrm{Cpd}^{\mathrm{b}}$} & \multirow[b]{2}{*}{ P-glycan type ${ }^{g}$} & \multicolumn{3}{|c|}{$\begin{array}{c}\text { Batch A } \\
\text { Relative intensity }\end{array}$} & \multicolumn{3}{|c|}{$\begin{array}{c}\text { Batch B } \\
\text { Relative intensity }\end{array}$} \\
\hline & & & $\operatorname{Avg}^{d}$ & $\mathrm{SE}^{\mathrm{e}}$ & $\begin{array}{l}\text { Newly } \\
\text { ID }^{f}\end{array}$ & $\operatorname{Avg}^{d}$ & $\mathrm{SE}^{\mathrm{e}}$ & $\begin{array}{c}\text { Newly } \\
\text { ID }^{f}\end{array}$ \\
\hline 1394.3655 & 5_2_0_0_0_2 & $b i-\mathrm{p}-\mathrm{HM}$ & 0.39 & 0.03 & & 0.52 & 0.08 & \\
\hline 1556.4190 & 6_2_0_0_0_2 & $b i-p-H M$ & 4.12 & 0.48 & & 4.77 & 0.21 & \\
\hline 1718.4720 & 7_2_0_0_0_2 & $b i-p-H M$ & 23.29 & 0.36 & & 20.03 & 0.73 & \\
\hline 1880.5241 & 8_2_0_0_0_2 & $b i-\mathrm{p}-\mathrm{HM}$ & 5.59 & 0.58 & & 5.99 & 0.38 & \\
\hline 2042.5745 & 9_2_0_0_0_2 & $b i-\mathrm{p}-\mathrm{HM}$ & 0.67 & 0.06 & & 0.80 & 0.03 & \\
\hline 1152.3457 & 4_2_0_0_0_1 & mono-p-HM & 1.10 & 0.13 & & 1.67 & 0.17 & \\
\hline 1314.4004 & 5_2_0_0_0_1 & mono-p-HM & 7.10 & 0.23 & & 9.07 & 0.11 & \\
\hline 1476.4529 & 6_2_0_0_0_1 & mono-p-HM & 19.29 & 0.12 & & 18.57 & 0.46 & \\
\hline 1638.5064 & 7_2_0_0_0_1 & mono-p-HM & 19.41 & 0.44 & & 17.27 & 0.30 & \\
\hline 1800.5592 & 8_2_0_0_0_1 & mono-p-HM & 0.45 & 0.08 & & 0.44 & 0.09 & \\
\hline 1962.6071 & 9_2_0_0_0_1 & mono-p-HM & - & - & & 0.12 & 0.04 & 0 \\
\hline 1679.5304 & 6_3_0_0_0_1 & p-hybrid & 1.46 & 0.11 & & 1.99 & 0.47 & \\
\hline 1759.5011 & 6_3_0_0_0_2 & p-hybrid & - & - & & 0.12 & 0.03 & ० \\
\hline 1841.5855 & 7_3_0_0_0_1 & p-hybrid & 4.20 & 0.24 & & 5.39 & 0.19 & \\
\hline 1921.5502 & 7_3_0_0_0_2 & p-hybrid & - & - & & 0.10 & 0.01 & 0 \\
\hline 2044.6561 & 7_4_0_0_0_1 & p-hybrid & 0.13 & 0.06 & & 0.16 & 0.01 & 0 \\
\hline 2124.6328 & 7_4_-0_0_0_2 & p-hybrid & - & - & & 0.10 & 0.02 & 0 \\
\hline 2286.6798 & 8_4_0_0_0_2 & p-hybrid & - & - & & 0.07 & 0.02 & 0 \\
\hline 2132.6800 & 7_3_0_1_0_1 & p-hybrid & 12.28 & 0.32 & & 12.72 & 0.67 & \\
\hline 1728.6045 & 5_3_0_1_0_0 & Non-p & 0.22 & 0.03 & & 0.07 & 0.00 & \\
\hline 910.3234 & 3_2_0_0_0_0 & Non-p & 0.10 & 0.01 & & - & - & \\
\hline 1072.3784 & 4_2_0_0_0_0 & Non-p & 0.19 & 0.03 & & - & - & \\
\hline
\end{tabular}

g Phosphorylated glycan types, $b i$-p-HM=bi-phosphorylated high mannose; mono-p-HM=mono-phosphorylated high mannose; P-hybrid=phosphorylated hybrid type; Non-p=non-phosphorylated glycan 


\begin{tabular}{|c|c|c|c|c|c|c|c|c|}
\hline \multicolumn{9}{|c|}{ The third eluent by $40 \% \mathrm{ACN}$ in $\mathrm{H}_{2} \mathrm{O}$ with $0.05 \% \mathrm{TFA}^{\mathrm{a}}$} \\
\hline \multirow[b]{2}{*}{ Mass } & \multirow[b]{2}{*}{$\mathrm{Cpd}^{\mathrm{b}}$} & \multirow[b]{2}{*}{ SIA type ${ }^{h}$} & \multicolumn{3}{|c|}{$\begin{array}{l}\text { Batch A } \\
\text { Relative intensity }\end{array}$} & \multicolumn{3}{|c|}{$\begin{array}{l}\text { Batch B } \\
\text { Relative intensity }\end{array}$} \\
\hline & & & $\operatorname{Avg}^{d}$ & $\mathrm{SE}^{\mathrm{e}}$ & $\begin{array}{l}\text { Newly } \\
\text { ID }^{f}\end{array}$ & $\operatorname{Avg}^{d}$ & $\mathrm{SE}^{\mathrm{e}}$ & $\begin{array}{c}\text { Newly } \\
\text { ID }^{f}\end{array}$ \\
\hline 3025.0668 & 6_5_1_3_0_0 & 3 NeuAc & 36.93 & 0.78 & & 37.10 & 0.94 & \\
\hline 3390.2007 & 7_6_1_3_0_0 & $3 \mathrm{NeuAc}$ & 0.58 & 0.36 & 0 & 2.01 & 0.13 & 0 \\
\hline 2222.7858 & 5_4_0_2_0_0 & $2 \mathrm{NeuAC}$ & 13.05 & 0.49 & & 11.48 & 0.22 & \\
\hline 2368.8429 & 5_4_1_2_0_0 & $2 \mathrm{NeuAc}$ & 20.31 & 0.33 & & 20.46 & 0.80 & \\
\hline 2587.9131 & 6_5_0_2_0_0 & $2 \mathrm{NeuAc}$ & 0.70 & 0.16 & 0 & 0.75 & 0.16 & 0 \\
\hline 2733.9730 & 6_5_1_2_0_0 & $2 \mathrm{NeuAc}$ & 11.45 & 0.52 & & 11.78 & 0.76 & \\
\hline 3099.0996 & 7_6_1_2_0_0 & $2 \mathrm{NeuAC}$ & 0.42 & 0.16 & & 0.48 & 0.00 & \\
\hline 1566.5534 & 4_3_0_1_0_0 & $1 \mathrm{NeuAc}$ & 0.19 & 0.01 & 0 & 0.17 & 0.02 & \\
\hline 1712.6128 & 4_3_1_1_0_0 & $1 \mathrm{NeuAc}$ & 0.48 & 0.01 & O & - & - & \\
\hline 1915.6932 & 4_4_1_1_0_0 & $1 \mathrm{NeuAc}$ & 0.15 & 0.00 & 0 & - & - & \\
\hline 1931.6876 & 5_4_0_1_0_0 & $1 \mathrm{NeuAc}$ & 3.89 & 0.47 & & 3.29 & 0.36 & \\
\hline 2077.7443 & 5_4_1_1_0_0 & $1 \mathrm{NeuAc}$ & 6.50 & 0.26 & & 7.03 & 0.61 & \\
\hline 1890.6602 & 6_3_0_1_0_0 & $1 \mathrm{NeuAc}$ & 0.48 & 0.05 & & 0.41 & 0.11 & \\
\hline 2442.8791 & 6_5_1_1_0_0 & $1 \mathrm{NeuAc}$ & 1.49 & 0.14 & & 1.25 & 0.17 & \\
\hline 2132.6804 & 7_3_0_1_0_1 & $1 \mathrm{NeuAc}$ & 1.99 & 0.38 & & 2.04 & 0.20 & \\
\hline 2384.8358 & 5_4_1_1_1_0 & $1 \mathrm{NeuAc} / 1 \mathrm{NeuGc}$ & 0.28 & 0.02 & & 0.30 & 0.03 & 0 \\
\hline 2749.9680 & 6_5_1_1_1_0 & $1 \mathrm{NeuAc} / 1 \mathrm{NeuGc}$ & 0.12 & 0.00 & 0 & 0.11 & 0.01 & 0 \\
\hline 1259.4663 & 3_3_1_0_0_0 & O NeuAc & 0.09 & 0.05 & & - & - & \\
\hline 1421.5135 & 4_3_1_0_0_0 & O NeuAc & 0.11 & 0.05 & & 0.15 & 0.07 & \\
\hline 1640.5898 & 5_4_0_0_0_0 & O NeuAc & 0.34 & 0.11 & & 0.37 & 0.06 & \\
\hline 1786.6505 & 5_4_1_0_0_0 & O NeuAc & 0.27 & 0.18 & & 0.64 & 0.09 & \\
\hline 1841.5845 & 7_3_0_0_0_1 & O NeuAc & 0.17 & 0.01 & & 0.21 & 0.06 & \\
\hline
\end{tabular}

${ }^{\mathrm{h}}$ Sialylated glycan types (including the degree of sialylation) 